\section{Global Proceedings Repository \\ American Research Foundation}

ISSN 2476-017X

Available online at http://proceedings.sriweb.org

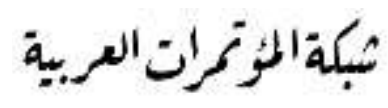

http://arab.kmshare.net/

ARF

The 10th International Scientific Conference

Under the Title

"Geophysical, Social, Human and Natural Challenges in a Changing Environment"

$$
\begin{aligned}
& \text { المؤتمر العلمي الدولي العاشر } \\
& \text { تحت عنوان "التحديات الجيوفيزيائية والاجتماعية والانسانية والطبيعية في بيئة متغيرة" } \\
& 26 \text { - } 25 \text { يوليو - تموز } 2019 \text { - اسطنبول - تركيا }
\end{aligned}
$$

http://kmshare.net/isac2019/

\title{
Israeli Media Information Impact on the Identity of Palestinian Press Release Employed by Palestinian News Websites
}

\author{
Ma'an news agency as sample \\ Yousef Shakarnah \\ PTUK University, Al Arob, Hebron, Palestine \\ Yousef.shakarnah@ptuk.edu.ps
}

\begin{abstract}
This study aims to monitor the mechanism followed by Palestinian news websites dealing with news in the Israeli media especially websites, and how this data is sorted to serve the goals of Palestinian websites and citizens. The main hypothesis in the study deals with transforming data from Israeli media as is without any professional review. This makes Palestinian news websites a spokesperson to Israeli media repeating their idioms and terms that prejudice the identity and message of Palestinian media.

The power of the Israeli media and its concern in competition and acceleration contributed in getting the Palestinian websites out of their goals of supporting the affairs of the Palestinians and the Palestinian cause to confront the Israeli occupation. The study responds to these questions: Do the Palestinian websites become a platform to rebroadcast the Israeli news? Do these websites serve the Israeli media message? Does the competition between these websites is a cause to follow the Israeli speech? The researcher will apply the descriptive approach based on interviewing the staff of news websites specialized in Israeli media due to the lack of studies in this area.
\end{abstract}




\section{Global Proceedings Repository \\ American Research Foundation}

ISSN 2476-017X

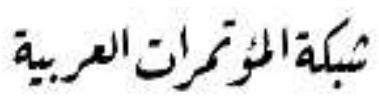

http://arab.kmshare.net/

Available online at http://proceedings.sriweb.org

It includes terms related to the Palestinian cause mainly the Israeli media, the Palestinian news websites, and the Israeli version. In order to achieve the goal of the study, the researcher divided the study into two divisions; First: the history of Israeli media and the evolution of the Palestinian new websites. This section deals with two subjects; the history of Israeli media, and the formation and evolution of the Palestinian new websites. The second division searches the sources of Israeli information and how the Palestinian news websites handle it. This section discusses two subjects; the Israeli information sources, and the Palestinian news websites handling with the Israeli media. These divisions aim to reach the goal of the study and come out with results valid for circulation.

Keywords: Israeli, news. websites, Identity, Palestine

$$
\begin{aligned}
& \text { أثر استخدام المواقع الإخبارية الفلسطينية معلومات الإعلام الاسرائيلي على } \\
& \text { هوية الخبر الفلسطيني } \\
& \text { وكالة معا الاخبارية نموذجا المبريا } \\
& \text { يوسف شكارنة } \\
& \text { جامعة فلسطين التقنية } \\
& \text { " يشكر الباحث جامعة فلسطين التقنية على دعمها اتمام ونشر هذا البحث" }
\end{aligned}
$$

تكمن أهمية بحث "تعامل المواقع الاخبارية الفلسطينية مع معلومات الإعلام الاسرائيلي" في رصد الآلية

التي تتبعها تلك المواقع مع مختلف الأخبار الواردة في وسائل الإعلام الاسرائيلي من إعلام مرئي ومسموع وخاصة

الالكتروني، وكيفية فرز هذه المعلومات بما يخدم أهداف المواقع الفلسطينية والمواطن الفلسطيني.

وتقوم الفرضية الرئيسية في البحث أن المواقع الاخبارية الفلسطينية أمام تدفق سيل الاعلام الاسرائيلي

وحرصها على المنافسة والسرعة ساهم بثكل رئيسي في خروج تلك المواقع عن أهدافها التي تأسست من أجلها في

دعم قضايا المواطن الفلسطيني والقضية الفلسطينية في مواجه الاحتلال الإسرائيلي.

وسيجيب البحث عن التساؤلات الرئيسية والتي تتمثل في: هل اصبحت المواقع الفلسطينية منبرا لاعادة بث

الاخبار الاسرائيلية؟ وهل أصبحت تلك المواقع بالتالي تخدم الرسالة الاعلامية الاسرائيلية؟ وهل التتافس بين تلك

المواقع شكل مبررا لأن تصبح تابعاً للخطاب الاسرائيلي؟ 


\section{Global Proceedings Repository \\ American Research Foundation}

ISSN 2476-017X

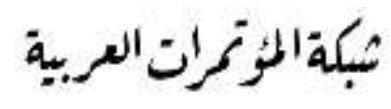

http://arab.kmshare.net/

Available online at http://proceedings.sriweb.org

وسيقوم الباحث باستخدام أسلوب البحث الوصفي الذي يعتمد على إجراء المقابلات للعاملين في المواقع

الاخبارية الفلسطينية مختصين في الإعلام الإسرائيلي، خاصة في ظل نقص الدراسات في هذا المجال.

ويضم البحث عددا من المفاهيم المرتبطة بجوانب القضية وأهمها: الإعلام الإسرائيلي، المواقع الاخبارية

الفلسطينية، الرواية الاسرائيلية.

ومن أجل تحقيق هدف الدراسة تتقسم الى جزئين، الاول: "تاريخ الاعلام الاسرائيلي وتطور المواقع

الاخبارية الفلسطينية". وينقسم الجزء الأول إلى مبحثين، الأول يتتاول تاريخ الإعلام الإسرائيلي، والثاني يتحدث عن

نشأة وتطور المواقع الاخبارية الفلسطينية.

وفي الجزء الثاني من البحث تحت عنوان "مصادر المعلومات الإسرائيلية وتعامل المواقع الاخبارية

الفلسطينية معها" وسيقوم الباحث تقسيمه إلى مبحثين، الأول: مصادر المعلومات الاسرائيلية والثاني يناقش تعامل المواقع الاخبارية الفلسطينية مع الإعلام الإسرائيلي، وتأتي هذه الأجزاء من اجل الوصول الى هدف الدراسة والخروج بنتائج تكون صالحة للتعميم.

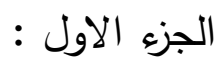

تاريخ الاعلام الاسرائيلي وتطور الإعلام الفلسطيني

الفصل الأول: التطور التاريخي للاعلام الاسرائيلي:

نثأت الصحافة الإسرائيلية قبل قيام الدولة العبرية, وبدأت عملها الى جانب القيادة اليهودية التي قادت

نشاط الفصائل العسكرية والسياسية, وعند إعلان دولة إسرائيل عام 19 1 إنانت أول خطوة قامت بها حكومة بن غوريون هي تحويل إذاعة صوت القدس الى اذاعة اسرائيل, وأفرزت في تلك الفترة ما عرف بـ " هيئة رؤساء تحرير الصحف" والتي يعتقد أنها جزء من جهاز الاستخبارات الاسرائيلي "الموساد", لان استمرارها يؤكد على أهمية دورها

المعروف والدحدد الذي يحول دون انهائها, وشكلت الهيئة دورا أشبه ما يكون بالرقيب على الصحافة الاسرائيلية نفسها, لخدمة مصالح السلطة, وهذا يبرز التتاقض في حرية التعبير والصحافة في أي مجتمع ديمقراطي. وتتمثل آلية عمل الهيئة في تلقي المعلومات من كبار المسؤولين, كرئيس الحكومة ورؤساء الاجهزة الامنية, وضباط الجيش حول ما يراد نشره بالدرجة الاولى, كأن يتم اطلاعهم على وقوع حدث معين, أو قرار , بحيش يتحاشون الاشارة اليه من قريب أو بعيد.1 


\section{Global Proceedings Repository \\ American Research Foundation}

ISSN 2476-017X

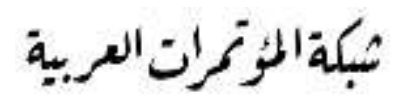

http://arab.kmshare.net/

Available online at http://proceedings.sriweb.org

وتتعرض الهيئة اليوم لانتقادات شديدة, وتعتبر ظاهرة غير مستحبة في الاعلام الاسرائيلي لانها تثكل قوة

ضاغطة تحول دون حرية التعبير, ويؤمن معارضوها بأن نشوئها كان لخدمة المصالح الصهيونية, واخفاء الاسرار وتحركات المنظمات العسكرية, وقد تلاشى هذا الدور الان.

$$
\text { وظيفة الإعلام الإسرائيلي: }
$$

يكاد يكون من المستحيل إدراك أبعاد العمل الاعلامي الاسرائيلي دون الأهداف القومية الاسرائيلية العامة, وكذلك ما يتفرع عنها من أهداف خاصة في القارات والأقطار المختلفة, والتلازم الكامل القائم بين العمل الإعلامي الإسرائيلي وتلك الاهداف, وقد حدد البند الثالث من قانون الاذاعة والتلفزيون الذي وافق عليه الكنيست الاسرائيلي في آذار 970 ا و وظيفة الاذاعة والتلفزيون كالتالي, وهي نفس الوظيفة التي تطبق على باقي الوسائل بشكل أو بآخر : 1- بث البرامج التعليمية والتسلية والمعلومات في المجالات السياسية والاجتماعية والاقتصادية والثقافية والعلمية

$$
\text { والفني, بحيث تهدف الى: }
$$

ا ـ إبراز طابع الكيان اليهودي وانجازاته.

r. تتمية الصلة بالتقاليد اليهودية وتعميق معرفتها.

r. إبراز نمط حياة وثقافة الجماعات اليهودية في العالم.

2- بث برامج باللغة العربية, تمهيدا أرضية التفاهم المستقبلي مع الدول المجاورة المستخدمة للعربية, وذلك

$$
\text { حسب الأهداف الأساسية للدولة. }
$$

$$
\text { 3- بث برامج موجهة لليهود الخارج, وبرامج خارج حدود الدولة.2 }
$$

قد نظر الإعلام الإسرائيلي الى الصراع العربي الإسرائيلي منذ بداياته الاولى من منظار أمني, وكثيرا ما كان يكتفي بما تمليه عليه السلطة والأجهزة الأمنية من معلومات, مستخدمة ذلك في التظليل في المعلومات. 


\section{Global Proceedings Repository \\ American Research Foundation}

ISSN 2476-017X

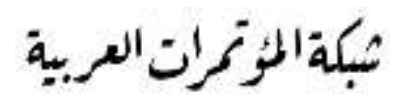

http://arab.kmshare.net/

Available online at http://proceedings.sriweb.org

وقد انتهج الإعلام الإسرائيلي منذ بداية الصراع الفلسطيني الإسرائيلي مبادئ أساسية في حديث تغطيته

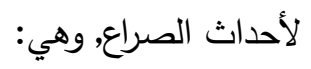

1-اللمسة الانسانية, من حيث ذكر الآباء والأطفال اليهود مما يضفي الطابع الإنساني والثخصي على "الإرهاب الفلسطيني" الذي تواجهه اسرائيل كل يوم.

2-السؤال البلاغي, حتى المؤيدين للفلسطينيين يواجهون صعوبة في الإجابة على الأسئلة الموجهة إليهم, حيث

انتهج المتحدثون الإعلاميون الاسرائيليون طرح المزيد من الأسئلة البلاغية الغير قابلة للإجابة كجزء من

$$
\text { جهودهم الإعلامية. }
$$

3-الإعتراف بوجود ثقافي بين الإسرائيليين والفلسطينيين, لكي يجد الغربيون أنفسهم قربيين من الاسرائيليين, نظرا

لاشتراكهم في الثقافة نفسها معهم وكذلك التقاليد والقيم. 3

وقد رأى المحررون في وسائل الإعلام الاسرائيلية أن التعددية الفكرية و الاختلافات الأيديولوجية بينهر,

يجب أن لا تشكل عائقا أمام تجنيد وسائل الإعلام بشكل تام, وهو تجنيد الوطن القومي اليهودي في فلسطين, لخدمة الهدف الاستراتيجي الأعلى, وبالتالي فإن المتمعن في هذه الوسائل منذ نشأتها يرى أن النظرة لطرفي الصراع الآخر "الثعب العببي الفلسطيني" لم تكن تختلف بين التيارات الفكرية والعقائدية المتصارعة اختلافاً جديرة بالانتباه والتسجيل.4

$$
\text { أدوات الإعلام الإسرائيلي: }
$$

يوجد في إسرائيل سلطة البث الرسمي, التي تؤطر في داخلها الإذاعة والتلفزيون والعبرية, ويشرف عليها هيئة مكونة من برلمان مؤلف من اب عضو, ثلاثون منهم من قبل الحكومة ورئيس الدولة, وهم يمثلون الأحزاب والتيارات السياسية المختلفة والثخصيات العامة, اما العضو الحادي والثلاثون تعيينه الوكالة اليهودية, وتهدف هذه اللجنة إلى اختيار اللجنة التتفيذية المؤلفة من سبعة أشخاص, وهم يمثلون أيضا التيارات السياسية المختلفة, لأن وجود التيارات 


\section{Global Proceedings Repository \\ American Research Foundation}

ISSN 2476-017X

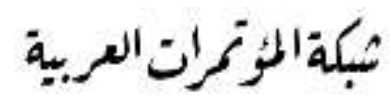

http://arab.kmshare.net/

Available online at http://proceedings.sriweb.org

المتصارعة في هذا الإطار تساعد في خلق توازن بين الضغوطات السياسية التي تمارس على العاملين في وسائل الإعلام الرسمية, ثم تكلف الحكومة أحد الاعلاميين المتخصصين المهنيين وذوي الخبرة, لتبوء منصب المدير العام للهيئة, ومدة خدمة الهيئة ثلاث سنوات غير قابلة للتجديد, والهدف من تحديد المدة منح الإذاعة والتلفزيون قسطا كبيرا من حرية عرض مختلف الآراء والاتجاهات.5 وتتلخص وظيفتها بوضع الاتجاه العام لبرامج البث التي تقوم بها الاذاعة والتلفزيون بكلا اللغتين العبرية والعربية.

$$
\text { الإعلام الإلكتروني في إسرائيل: }
$$

قررت الحكومة الاسرائيلية في الأول من يوليو r . . إستخدام مواقع الإنترنت الحكومية كوسائل إعلام

عبر الشبكة الاككترونية, وأصدرت تعليمات للمديرين العاملين في الوزارات بإخال مواد إعلامية على المواقع الخاصة وزاراتهر, تحمل مضامين تساهم في شرح السياسة الإسرائيلية مع ربطها بموقع وزارة الخارجية ورئاسة الحكومة, على أن يكون تحديد مضامين هذه المواد ضمن صلاحيات مكتب رئيس الحكومة ووزراء الدفاع, وسيتم نشرها باللغتين العبرية والإنجليزية, على أن يتم نشرها في وقت لاحق بلغات أخرى, ومنها اللغة العربية, وبالفعل هذا ما حدث بعد

2- مواقع إخبارية: موقع صحيفة يديعوت أحرونوت, موقع واللا العبري, صحيفة إسرائيل هيوم، القناة السابعة

الإسرائيلية, والثبكة الإخبارية العبرية 0404.

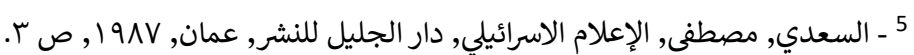

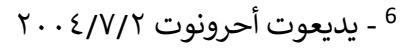




\section{Global Proceedings Repository American Research Foundation}

ISSN 2476-017X

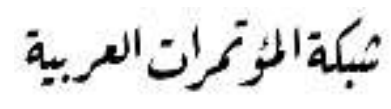

http://arab.kmshare.net/

Available online at http://proceedings.sriweb.org

$$
\text { طبيعة وخلفيات المواقع الإخبارية الإسرائيلية: }
$$

عند تقييم وسائل الإعلام الإسرائيلية يجب التفريق عندما يكون الأمر يتعلق بالثأن الإسرائيلي الداخلي أو بالثأن الفلسطيني، ففي الشأن الإسرائيلي وسائل الإعلام الإنسائيلية أكثر حرية وديموقراطية، ولا يوجد لها خطوط حمراء حتى لو وصل الأمر لأعلى المستويات السياسية، ومن هذه الوسائل من يدعم اليمين الإسرائيلي بثكل جلي وواضح مثل صحيفة إسرائيل هيوم، والقناة السابعة الإسرائيلية والثبكة الإخبارية العبرية 0404، ولكن هذا لا يعني تصنيف بقية وسائل الإعلام الإسرائيلية بأنها يسارية بل هي مفتوحة أكثر تجاه نقد الحكومة الإسرائيلية مثل القناة العاشرة الإسرائيلية والقناة الثانية الإنسرائيلية.

ولا يوجد في دولة الاحتلال الإسرائيلي صحف موالية للحكومة وصحف معارضة لها بالمفهوم التقليدي، الصحافة الإنرائيلية تدعم توجهات سياسية بالدرجة الأولى، بمعنى من يؤيد ويدعم نتتاهو أو حزب الليكود يدعمه سياسياً وإعلاميا سواء كان في الحكومة أو في المعارضة.

صحيفة هآرتس العبرية تختلف عن نهج الإعلام الإسرائيلي بشكل عام، وتجد فيها الرواية الفلسطينية حاضرة

في كثير من الأحداث، وحتى هناك من يكتب فيها ضد الاحتلال الإسرائيلي وعنصريته وجرائمه مثل الصحفي "جدعون ليفي" والصحفية "عميره هس".

لكن عندما يتعلق الأمر بالشأن الفلسطيني يتحول الإعلام الإنرائيلي (باستثناء صحيفة هآرتس إلى حدما) لإعلام عسكري وأمني بامتياز، ولا تجد فيه حضور إلا للرواية الأمنية الإسرائيلية الصادرة عن الناطق باسم جيش الاحتلال الإسرائيلي أو الناطق باسم شرطة الاحتلال الإسرائيلي.

بمعنى آخر في الشأن الفلسطيني يتحول الصحفي الإسرائيلي إلى جندي في جي الاحتلال الإسرائيلي ولكن

بزي مدني، وبدلاً من بندقة إل (إم 16) يحمل ميكروفون وكاميرا.7

كما ان هذه الوسائل متتوعة تتوع المجتمع السياسي الاسرائيلي او متتوعة بتتوع المجتمع الاسرائيلي سياسيا 7 - ضراغمة, محمد, متخصص في الإعلام الإسرائيلي, مقابلة بتاريخ .5/2/2018 


\section{Global Proceedings Repository \\ American Research Foundation}

ISSN 2476-017X

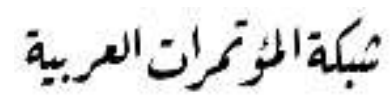

http://arab.kmshare.net/

Available online at http://proceedings.sriweb.org

وثقافيا وفنيا ومنها الدينية ومنها اليسارية وكذلك اليمينية المتطرفة ومنها المستقلة ومنها من يتجه نحو مثل موقع واللا المقرب لنتتياهو وكان محسوبا في فترة سابقة على صحيفة هآرتس فهو كان لملكية أحد كبار الأثرياء الإسرائيليين شديدي التأييد والتأثر بنتياهو.8 الفصل الثاني: تطور الإعلام الفلسطيني أصبحت المواقع الاخبارية في الواقع المعاصر شأنا مهما في حياة الناس أفراد ومجتمعات وتعددت هذه

المواقع والأساليب التكنولوجية التي تستخدمها وتختلف أنواع مضامينها, فلم يعد الإنسان يملك فكاكاً من وصوله اليها, وتأثيرها عليه, واخذ المعلومات سواء أكانت صحيحة أم خاطئة, حقيقة او فبركة, بحيث أصبحت تنتشر هذه المعلومات بسرعة بحيث لم يعد بالإمكان اللحاق بها.

ومن الملاحظ أن التطور الذي حدث ولا يزال يحدث في وسائل الإتصال والإعلام, يضيف باستمرار أدوات ووسائل جديدة, لكنها دائماً لا تلغي الوسائل السابقة عليها, بل تعيد كل وسيلة صناعة موقع جديد وطريقة جديدة في التأثير , مما ينبئ بأن خط التطور سيبقى متواصلاً, وهو ما يدعونا أن نواكب بإستمرار هذا التطور واستثمار وسائله. تتعدد الوسائل الاعلامية في فلسطين ويتنوع خطابها بناء على عدة عوامل, وتعدد البيئة التي ظهرت فيها هذه الوسائل الاعلامية هي السبب الأساسي وراء التتوع في الخطاب. بمعنى اخر , ان اختلاف الخطاب الإعلامي من وسيلة أو مؤسسة إلى اخرى نابع بالدرجة الاولى من اختلاف المشاريع السياسية التي نشأت في كنفها وتعبر عنها هذه المؤسسة أو تلك.

وتقسم هذه الوسائل الى عدة أقسام: الإعلام الحزبي الفصائلي, الإعلام الحكومي, الإعلام الخاص

والمستند في تأسيسه على رأس المال المحلي للاستثمار بالإضافة إلى الإعلام الممول أجنبيا. تعتبر كل مؤسسة عن خطاب مشروعها السياسي التي انبثقت منه, ويبدو ذلك متجليا في مستويات عدة, وأوضحها اختلاف المفردات 


\section{Global Proceedings Repository \\ American Research Foundation}

ISSN 2476-017X

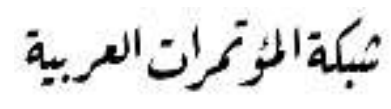

http://arab.kmshare.net/

Available online at http://proceedings.sriweb.org

والمصطلحات المستخدمة لوصف حدث ما أو طريقة صياغة الخبر , عدا عن الاختلاف في أولويات الأخبار من

حيث أهميتها والموضوعات والمساحات وصناعة القادة والمؤثرين.9

وقد نشأت الكثير من هذه الوسائل الاعلامية الفلسطينية في بيئة هيمن عليها افرازات خطاب

اتفاقيات أوسلو, التي حددت دور الإعلام بالعلاقة مع السلطة في سياق عملية "بناء المؤسسات" و "المحاسبة ومكافحة الفساد والمساءلة. كما استدخلت في هذه البيئة الإعلامية مفاهيم مثل "السلطة الرابعة" المعدول به في الدول الليبرالية والديمقراطية. وكانت هذه البيئة إحدى تجليات ونتائج تحول حركة التحرر الوطني الفلسطيني إلى سلطة ومؤسسات سياسية, فتحول الإعلام المركزي للثورة الذي كان يحمل همة التعبئة والحديث باسم الثثرة الى هيئة إذاعة وتلفزيون رسمية, وذراع إعلامي لحركة فتح واللجنة المركزية لها.

أما في فلسطين المحتلة عام 19 1 وتتكون البيئة الإعلامية من الإعلام الحزبي (الحركة

الاسلامية, التجمع, الجبهة..) , والإعلام الخاص الرأسمالي, بالإضافة إلى ظاهرة لافتة وهي انتشار مواقع إعلامية محلية للقرى والبلدات, تتقل وتغطي هذه المواقع الاحداث المحلية وتعزز خلق هويات فرعية. كما أن هناك ظاهرة آخذة بالانتشار وهي الإذاعات التي تبث من الخليل وجبل الخليل, من انشاء أهالي النقب, موجهة الى أهالي النقب. ويعتمد الخطاب الإعلامي في فلسطين المحتلة عام ^§ 19 بشكل عام على الخطاب القانوني, مع التركيز على مواضيع العنصرية, وغياب المساواة مع المستوطنين. وبعض المؤسسات والمواقع تتعامل مع ما يجري في الضفة والقدس والقطاع على أنه يجري في "دولة اخرى". مثل تخصيص بعض المواقع زاوية " فلسطينيات" للحديث عما يجري في الضفة والقطاع. وكأن ما يجري في الناصرة وأم الفحم مثلا ليس فلسطيني.

وبالرجوع الى البيئة الاعلامية في الضفة, فإن وسائل الإعلام الرسمية المستندة إلى رأس المال الخاص, و المستقيد من التمويل الأجنبي, تبنت هي كذلك نهج خطاب "سلطة المؤسسات", وحاولت لعب دور

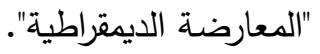

9 ـ الأداء الإعلامي الفلسطيني خلال الهبة الشعبية, ورقة عمل, دائرة سليمان الحلبي .20/11/2011 


\section{Global Proceedings Repository \\ American Research Foundation}

ISSN 2476-017X

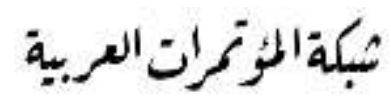

http://arab.kmshare.net/

Available online at http://proceedings.sriweb.org

اما الفصائل الفلسطينية فقد اعتمدت في وسائلها الاعلامية على بث الخطاب الحزبي الموجه

أساسا نحو جمهورها, عدا عن بث المناكفات السياسية مع بقية الفصائل. وتعاني معظم المؤسسات الإعلامية الحزبية

من فثل في خلق حالة نقد ذاتي داخل جمهرها, فالجمهور يحدد تفاعله مع الرسالة الاعلامية بالقبول او الرفض بالنظر إلي هويتها (اسم القناة او الموقع او الكاتب..). ومن ثم يتخذ هذا التفاعل طابع المناكفة والبحث عن الفضائح

وتتناقض المواقف عند "الخصوم" والتذكير بالتاريخ النضالي للفصيل, والكثير مما يطلق عليه في الثقافة السياسية الفصائلية "بالمزايدات". وعادة ما تغيب النقاشات الجادة والتي تفصل بين ما يقال من يقول, وبالنتيجة تغيب روحية النقد الذاتي ما بين جمهورها, ويبقى التحشيد وبناء الهوية الفصائلية هو سيد الموقف, عدا محاولة البحث عن أرضية

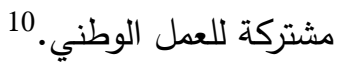

ولا يمكننا التطرق الى الخارطة والبيئة المشكلة للاعلام الفلسطيني دون التحدث عن صعود

الاجهزة الاعلامية العربية التي ارتبطت بمصالح وسياسات دول معينة أو فئات اجتماعية مختلفة, وقد شكلت شبكة

الجزيرة الاخبارية في بداية انطلاقتها نقلة نوعية في الجهاز الإعلامي العربي, فقد تسارع بعدها ظهور القنوات التلفزيونية مثل قنوات العربية والميادين والحدث والعالم وروسيا اليوم والحرة, وغيرها من القنوات التي تخدم سياسات الدول التي تمولها. وتباين مواقف وسياسات هذه الوسائل تبعا للسياسة الخارجية لهذه الدول وعادة ما تتعكس صراعاتها على سياستها الإعلامية في الساحة الفلسطينية وهي لاعب أساسي في الساحة الإعلامية المحلية. وقد تفاعلت هذه المؤسسات بطرق متفاوتة مع الهبة الشعبية, تراوحت ما بين: الدعم والاسناد

الكامل بما يتسق مع الموقف السياسي واجندات, والتبني الماكر الذي يريد متابعة الحدث بانتظار ما ستؤول إليه الأمور واتضاح الموقف السياسي لمن تمثله هذه الوسائل, مع بث رسائل اعلامية تحذر من طرق باب المجهول 


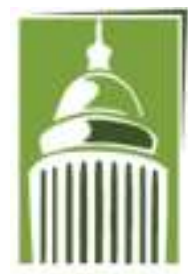

ARF
Global Proceedings Repository

American Research Foundation

ISSN 2476-017X

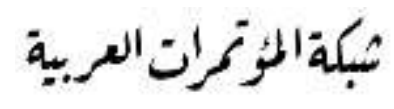

http://arab.kmshare.net/

Available online at http://proceedings.sriweb.org

وتذكر الدمار الذي حل بالمتمع الفلسطيني نتيجة للأفعال غير المدروسة "والتبني الاستثماري" كحدث إعلامي يشكل هماً واهتماماً للجمهور وفرصة لرفع عدد المتابعين.11 المواقع الاخبارية الفلسطينية

هنالك ضرورة التتويه انه لا يوجد أي أبحاث أو دراسات مخصصة حول نشأة المواقع الاخبارية الالكترونية الفلسطينية، ولكن كان لتطور وسائل التواصل والاتصال الفضل الأول في ضرورة تطوير وسائل الخطاب الإعلامي الفلسطيني، وصولا الى استخدام وسائل التواصل الاجتماعي الان كوسائل اعلامية تحصد جمهورا واسعا.12 - (12

المواقع الاخبارية الفلسطينية بدأت انطلاقتها فعليا منو منصات مواقع المنتديات التي كانت تأخذ دورا مهما في بداية الالفية الثالثة ومن ثم اجتياح المواقع الاجتماعية حياة الناس. والذي كان أول هذه المواقع هو موقع يعرب الاخباري في تسعينيات القرن الماضي. وحتى الآن لا يوجد قانون لترخيص الاعلام الآكتروني أيا كان شكله في فلسطين لمعرفة عددها وتتظيمها، وهناك اجتهاد داخل وزارة الإعلام الفلسطينية بهذا الخصوص وعليه، تم ترخيص 21 موقعا إلكترونيا حتى الآن، فيما يعمل على الساحة الفلسطينية عدد أكبر بكثير من هذا الرقم. وهي على النحو التالي: المواقع الإلكترونية المرخصة من قبل وزارة الإعلام: 12 - يونس, نداء, وزارة الإعلام الفلسطيني, مقابلة بتاريخ .6/2/2018 


\section{Global Proceedings Repository \\ American Research Foundation}

ISSN 2476-017X

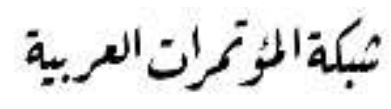

http://arab.kmshare.net/

Available online at http://proceedings.sriweb.org

\begin{tabular}{|c|c|c|c|}
\hline وكالة راية للأنباء & .12 & "ANB" المؤسسة العربية للاخبار & .1 \\
\hline وكالة فلسطين ؟؟ الاخبارية & .13 & فلسطين برس & .2 \\
\hline وكالة حياد الأخبارية & .14 & جمعية الرياضة في فلسطين "PAL SPORT" & .3 \\
\hline وكالة الاقصى الان الاخبارية & .15 & شبكة فلسطين الاخبارية & .4 \\
\hline وكالة ايلان الاخبارية & .16 & وكالة معا الاخبارية & .5 \\
\hline وكالة زاد نيوز الاخبارية & .17 & وكالة الصحافة الفلسطينية صفا & .6 \\
\hline وكالة أسوار برس الاخبارية & .18 & وكالة السلام الاخبارية & .7 \\
\hline وكالة ريادة الاخبارية & .19 & وكالة الحقيقة للأنباء & .8 \\
\hline وكالة الرواسي الاخبارية & .20 & فلسطين برس & .9 \\
\hline وكالة الاوائل الاخبارية & .21 & وكالة وطن للانباء & .10 \\
\hline & & وكالة آرام الإخبارية & .11 \\
\hline
\end{tabular}

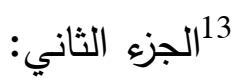

تأثير الإعلام الإسرائيلي وتعامل المواقع الاخبارية الفلسطينية معه الفصل الأول: تأثير الإعلام الإسرائيلي:

لا يمكن القول بأن الإعلام الاسرائيلي يؤثر على الإعلام الفلسطيني بمعنى ان الاعلام الفلسطيني ينقاد وراء الاعلام الاسرائيلي لكن هنالك ضرورات موضوعية, ميدانية تتعلق بالترابط القصري الذي يربط المجتمع الفلسطيني 


\section{Global Proceedings Repository \\ American Research Foundation}

ISSN 2476-017X

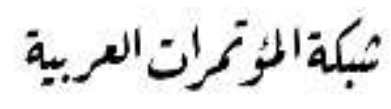

http://arab.kmshare.net/

Available online at http://proceedings.sriweb.org

الخاضع للاحتلال باسرائيل التي تحتل الفلسطينيين, وهي روابط او معطيات تتعلق بالحياة اليومية واخرى تتعلق بالوضع السياسي والاقتصادي الفلسطيني بعنى ان الفلسطينيين مجبرين على التعامل مع الإعلام الاسرائيلي.

يستقي الإعلام الفلسطيني المعلومات المتعقة بالعمليات الفلسطينية تحديدا التي تقع في المناطق الاسرائيلية

من المصادر الاعلامية الاسرائيلية بمعنى العمليات او الاهداف التي تكون التغطية فيها محصورة قصرا بالقوة العسكرية على الاعلام الاسرائيلي لمنع اي وسائل اعلامية فلسطينية أو غير فلسطينية من دخول هذه المواقع وبهذا يكون الاعلام الاسرائيلي مصدرا لا يوجد غيره للاعلام الفلسطيني.

الاعلام الفلسطيني لا ينقاد ويحاول ان يعادل هذه المعلومات مع معلومات بديلة لايضاح الصورة خاصة حين يتضح مكان منفذ العملية او موقع العملية بما يعادل الصورة.

الإعلام الفلسطيني في صور محاربته للانقياد نجح بصورة كبيرة لايجاد أو اشتقاق مصطلحات رديفة

المصطلحات التي يستخدما الاعلام الاسرائيلي حين ينقل الصورة او الخبر او نبا او معلومة عن هذا الإعلام بمعنى أن حتى الإعلام الإسرائيلي فشل في فرض مصطلحاته على الإعلام الفلسطيني لكن للضرورة أحكامها بهذا الثأن.14 - (14

ومع تطور الإعلام الفلسطيني وتعدد مصادره إن تأثير الإعلام الاسرائيلي في تراجع, لكن يمكن أن يكون له بصمات في بعض وسائل الإعلام الفلسطينية وليس في كافة وسائل الإعلام الفلسطينية. يوجد اليوم مهنية ما في المواقع الاخبارية الفلسطينية ذات البنية والطاقم الكامل, فأصبح هناك محللين واعلاميين يتعاملون مع المعلومة الاسرائيلية بحذر عالي وتحليل في هذه المواقع للتعرف على الدوافع وراءها. إن التأثير على الإعلام الفلسطيني بوجه عام, في المرحلة الراهنة أقل بكثير مع انه لا زال مصدرا مهما للعديد من الأخبار ذات الصلة بوجود الاحتلال لأن من الصعب جدا على الاعلام الفلسطيني أن يتزود بهذه المعلومات.

14 ـ اللحام, فؤاد, مدير قسم الاسرائيليات في وكالة معا الاخبارية, مقابلة بتاريخ .8/2/2018 


\section{Global Proceedings Repository \\ American Research Foundation}

ISSN 2476-017X

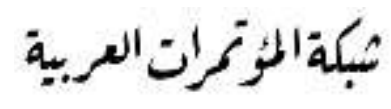

http://arab.kmshare.net/

Available online at http://proceedings.sriweb.org

المفارقات يجب أن تكون في طبيعة الناس التي تهتم في هذا الثأن وكيفية تحليل هذه البيانات وإظهارها للجمهور الفلسطيني, هذه الفروقات التي يمكن أن نجدها بين وسائل الإعلام المختلفة. هناك ضرورة للاعلام الفلسطيني من متابعة وسائل الإعلام الاسرائيلية ومتابعة كل ما يصدر عن اسرائيل لكن المهم في كيفية اخذ هذه المعلومات ونشرها عبر وسائل الإعلام الفلسطينية وهذا يحتاج الى مهنية عالية وقدرة تحليل هذه البيانات.15

غياب الرواية الفلسطينية في المواقع الإخبارية الإسرائيلية: "هدف الإعلام الإسرائيلي بالأساس, التقليل من شأن الفلسطينيين كثعب", أبرز نموذج على ذلك إستبدال عبارة الثعب الفلسطيني بعبارة الفلسطينين, ولكل من هاتين العبارتين مدلول كبير • وإن متابعة وسائل الإعلام الإسرائيلية يثير علامات سؤال حول مهنية ومصداقية مراسلين ومحررين وصحف, ليس فقط بما تقوله, بل بما لا تقوله, وتتتهج وسائل الإعلام الإسرائيلي بشكل عام اسلوب الترويج للموقف الرسمي ورواية الأحداث وعدم التطرق لمعاناة الفلسطينيين.

وبشكل مقصود تقوم المواقع الاخبارية الإسرائيلية بتصوير الفلسطينين وكأنهر مجرد أرقام, الثهداء يسقطون يوميا ويصاب العشرات من الفلسطينيين وغالبا يوجد هنالك عدد من الثهداء أو المصابين الاطفال, وتكتفي المواقع الإخبارية الإسرائيلية بنشر عدد التتلى والجرحي, وجميع هؤلاء الضحايا حسب هذه المواقع سقطو في مواجهات مع قوات الجيش او حملو اسلحة بيضاء بغض النظر انه يمكن السيطرة عليهم وعدم إطلاق النار نحوهم, وتعدد إغفال ذكر هذه الظروف. وحسب هذه المواقع انهم سقطوا عندما اطلقوا النار على على جنود او القوا الحجارة, أي انهم هم المتدون والجنود يدافعون عن أنفسه, ولا تذكر الاسماء ولا الأعمار ولا اماكن سكنهم ولا ظروف استشهادهم وعدم تقديم أي وجهة نظر فلسطينية. وهذا الامر لله علاقة بميزان التغطية لصالح الاسرائيلي, فإن تغطية "العنف" ان صح التعبير الذي يعني منه الطرفان غير متوازن, ولذلك نرى ان تغطية "عنف" الفلسطينيين أعلى من عنف الإسرائيلين 


\section{Global Proceedings Repository \\ American Research Foundation}

ISSN 2476-017X

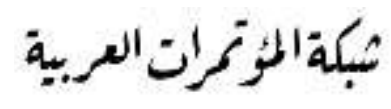

http://arab.kmshare.net/

Available online at http://proceedings.sriweb.org

على عدة مستويات, ويتم تزويد تفاصيل مسهبة ذات علاقة بالضحايا الإسرائيليين فيما لا تظهر تلك التفاصيل فيما يتعلق بالضحايا الفلسطينيين.16

لماذا يتجه الإعلام الفلسطيني للتزود من الإعلام الاسرائيلي؟

ان الاعلام الفلسطيني ليس جديدا انه ينظر الاعلام الاسرائيلى ويتابع القضايا والأخبار والتحليلات, ليست

ظاهرة جديدة. على العكس من ذلك في مرحلة تاريخية معينة, حتى على الصحف والجرائد اليومية في فترة الثمانينات العديد من الأخبار التي كانت موجودة كانت من الاعلام العبري وعند النظر الى ارشيف جريدة الثعب والقدس والفجر في الانتفاضة العديد من الأخبار كانت من الإعلام العبري.

يوجد فرق كبير اليوم فيوجد العديد من الوسائل الاعلامية الفلسطينية الخالصة وليس فقط المكتوبة ولكن أيضا المقروءة والمسموعة ويوجد إعلاميين فلسطينيين لليهم الخبرة والقدرة والمعلومات.

كون وجود الاحتلال على ارتباط مباشر مع الثعب الفلسطيني والصراع الدائم والمستمر يوجد تقاطع في الأخبار فالعديد من الأخبار التي يتتاولها الإعلام الفلسطيني جزء منها هو في الواقع في الجانب الاسرائيلي لذلك نجد الاعلام الفلسطيني ينظر للاعلام الاسرائيلي ويحاول أن يجد مصدر من داخل هذا الاعلام لكن الفرق ما بين الوسائل الاعلامية الفلسطينية المختلفة وفي كيفية النظر داخل الإعلام العبري , يوجد لدينا بعض المؤسسات الاعلامية تعتقد أن مصدر المعومة الصحيحة هو في الإعلام العبري ويتعامل مع هذا الاعلام على اساس انه معلومة نهائية ويجب التعامل معها كما هي, في الوقت الذي لدينا فيه مؤسسات اعلامية تنظر للاعلام العبري كونه موجه او "اعلام عدو" يوجد عليه رقابة عسكرية دائمة بالاضافة للرقابة الذاتية لوسائل الاعلام.17

يظهر في الاحداث الساخنة والحروب والاعمال العنيفة او عندما يشتد الصراع نجد كافة وسائل الاعلام الاسرائيلية وكأنها تتحدث بلغة واحدة وفقط لغة الجيش ولغة المخابرات ولغة الحكومة الاسرائيلية.

16 ـ قضايا إسرائيلية, مجلة, العدد 7, ص •V. 17 ـ عطية, ناصر, المحلل السياسي لوكالة معا الإخبارية, مقابلة بتاريخ .7/2/2018 


\section{Global Proceedings Repository \\ American Research Foundation}

ISSN 2476-017X

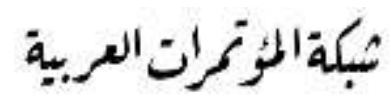

http://arab.kmshare.net/

Available online at http://proceedings.sriweb.org

الإعلام الفلسطيني يتجه للإعلام الإسرائيلي من أجل نقل وجهة النظر والرواية الإسرائيلية لتعريف المجتمع

الفلسطيني بما يقوله الإعلام ورجال السياسية تجاه الصراع الفلسطيني، وهذه خطوة جيده لفهم التوجهات العامة للمجتمع الإسرائيلي.

كما يتجه للإعلام الإسرائيلي لكون الكثير من الأحداث تقع في مناطق تحت الاحتلال والسيطرة الإسرئيلية ويمنع الصحفي من الفلسطيني من الوصول إليها، في الضفة الغربية هي المساحة الوحيدة المسموح فيها للصحفي

الفلسطيني التتقل، بالتالي معظم ما يجري داخل فلسطين المحتلة 1948 يكون مصدره وسائل الإعلام الإسرائيلية. 18 المخاطر السياسية من استخدام الفلسطينيين للبيانات الإسرائيلية

في المؤسسات التي تأخذ المعلومة والبيانات او الاخبار الاسرائيلية كونها حقيقة مطلقة, بالنظر الى هذا الجانب يوجد مخاطر هائلة في تثويه الفهم لدى الثعب الفلسطيني لهذا الإعلام كونه موجه في استخدام المفردات وفي استخدام الوصف.

الإعلام الإسرائيلي في الأحداث الحالية متفق على توصيف الحالة الفلسطينية بـ "الارهاب" ومتوافق على

الرواية التي تظهر من الجيش أو المخابرات في وصف أي عملية في داخل "اسرائيل" وحتى في الضفة الغربية, فنقلها بالطريقة أو البيانات التي تتشر في وسائل الإعلام الاسرائيلية كما هي وعلى أنها حقيقة, وهذا له مخاطر من خلال انعكاسها على وعي المجتمع الفلسطيني كما هي الحالة في الإعلام الفلسطيني عند يضخم هذه الاحداث واعطائها بعد غير حقيقي يشوه الحالة الفلسطينية.

لذا إن بالضرورة يجب التوازن في التعامل مع طبيعة الاخبار التي تظهر في وسائل الإعلام الاسرائيلي وكيفية

استخدامها بما يخدم مصالح الثعب الفلسطيني او بمعنى اخر عدم تثويه الوعي والثقافة الوطنية الفلسطينية.19 


\section{Global Proceedings Repository \\ American Research Foundation}

ISSN 2476-017X

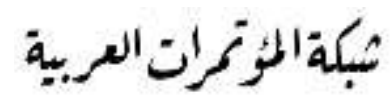

http://arab.kmshare.net/

Available online at http://proceedings.sriweb.org

الفصل الثاني: المواقع الاخبارية الفلسطينية والإعلام الإسرائيلي

وسائل الإعلام الفلسطينية تتقل في الكثير من الأحيان عن الإعلام الإنرائيلي لسببين، الأول: وهو أن

الأحداث تكون في منطقة تحت السيطرة الإسرائيلية الكاملة كما هو الحال في مدينة القدس الححتلة، والثاني: منع

جيش الاحتلال الإسرائيلي للصحفي الفلسطيني من الوصول لمكان الحدث للتغطية بحجة إنه منطقة عسكرية مغلقة.

إلا المهم في الأمر هو أن وسائل الإعلام الفلسطينية تشير في كثير من الأحيان للمصدر عندما يكون

المصدر من الإعلام الإسرائيلي كون الرواية الإسرائيلية رواية موجهة في العادة وتسعى لذذمة جيش الاحتلال

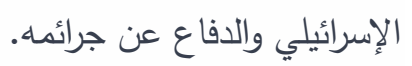

وكالتي معا وبي أن أن لايهم خاصية الترجمة من وسائل إعلام إسرائيلية ليس فقط في نقل الأخبار بل في

نقل وترجمة التحليلات العسكرية والسياسية والتي تكون بهدف التعريف بوجهة النظر الإسرائيلية.20

تعامل المواقع الاخبارية الفلسطينية مع الإعلام الإسرائيلي

هناك بعض وسائل الإعلام الفلسطينية تنقل الرواية الإسرائيلية بتجرد ونقل حرفي وهذه خطوة غير سليمة،

فعلى الإعلام الفلسطيني أن يعتد سياسية تحريرية عند النقل من الإعلام الإسرائيلي بحيث تعطى الرواية الإسرائيلية

عمق فلسطيني من خلال سرد معطيات فلسطينية تبين عدم صحتها إن كانت ملفقة أو فيها معطيات غير دقيقة، ولكن هذا لا يعني أن كل رواية إسرائيلية في الإعلام هي رواية ملفقة، مما يعني علينا التدقيق قبل النشر في أي رواية إسرائيلية.

من أهم الأمور التي تحتاج لتدقيق هي البيانات الصادرة عن الناطق باسم جيش الاحتلال الإسرائيلي والناطق باسم شرطة الاحتلال الإسرائيلي الذان يحاولان دائما إيجاد مبررات لعمليات "الإعدامات" للفسطينيين بدعوى 


\section{Global Proceedings Repository \\ American Research Foundation}

ISSN 2476-017X

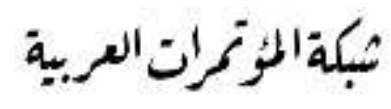

http://arab.kmshare.net/

Available online at http://proceedings.sriweb.org

الرد على هذه البيانات يكون من خلال التوضيح أن تتفيذ الفلسطيني لعملية ضد الاحتلال الإسرائيلي لا

يعني اعدامه، وفي معظم الحالات التي أعدم الاحتلال فيها فلسطينيين بدعوى تتفيذ عمليات طعن منذ بداية أحداث

أكتوبر 10 • r في الضفة والقدس والدخل المحتل كان بالإمكان اعتقال المنفذ أو المنفذة والسيطرة.21

احداث "الهبة الشعبية" منذ أكتوبر حتى نوفمبر 10 •

بدأت أحداث ما سمي بـ "الهبة الثعبية" في الاول من اكتوبر من عام 10 ـ ب تعود أسبابها وخلفياتها

المباشرة الى منع المصلين من الدخول إلى الحرم القدسي, والخشية الفلسطينيين من مخططات اسرائيلية لتقسيم

المسجد الاقصى زمانيا ومكانيا, كما تم التعامل مع الحرم الإبراهيمي في الخليل. وشملت الأحداث القدس ومناطق مختلفة داخل الخط الأخضر وفي مناطق الاشتباك الدائمة مع الحواجز الاسرائيلية في الضفة الغربية, وفي قلب مدينة الخليل, والحدود الإسرائيلية مع غزة.

يجب الاثارة الى ان ما يميز هذه الأحداث هو الانتقال السريع للتظاهر على جانبي الخط الأخضر من

الضفة والقطاع والمثلث والنقب والجليل ومناطق اخرى بمشاركة جيل من الثباب ممن لم يشاركو سابقا في مواجهة الاحتلال.

وتختلف هذه الأحداث احتجاجا على ممارسات الاحتلال في القدس باستخدام الاسلحة البيضاء, ووسائط

النقل وتحولها إلى ظاهرة بعدما كانت حالات فردية في السنوات السابقة من عمليات دهس وطعن للجنود

والمستوطنين. 22

الإعلام الفلسطيني خلال أحداث "الهبة الشعبية"

21

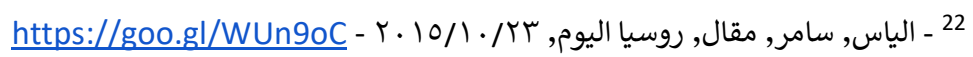




\section{Global Proceedings Repository American Research Foundation}

ISSN 2476-017X

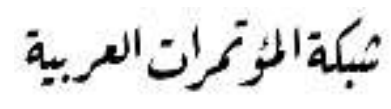

http://arab.kmshare.net/

Available online at http://proceedings.sriweb.org

وخلال هذه شهدت الاحداث، وتولت أجهزة الإعلام الإسرائيلي ترويج روايتها وتكفلت بإظهار المجتمع الإسرائيلي وكأنه في حالة دفاع عن النفس. وأخفق الإعلام الفلسطيني في إظهار الرواية الفلسطينية واعتمد في غالب الأحيان على الرواية الإسرائيلية، ما أدى لحالة من الفوضى في الإعلام المحلي.

واعتماد الإعلام الفلسطيني في كثير من الأحيان على مصادر معلومات عبرية دون التحقق أو التدقيق أو

دون محاولة البحث عن مصدر فلسطيني أتاح المجال لمثل هذه الفوضى, فالمسألة الأساسية هي أن "الرسالة الفلسطينية" ليست واضحة بالنسبة للفلسطينيين فكيف يوضحها الإعلام الفلسطيني للرأي العام الإسرائيلي والدولي. ومعظم الحالات التي نقلها الإعلام الفلسطيني عن العبري لا يستطيع الضحية الكلام، أي أنه قُتل، ما يعني إقفال القضية على الرواية الإسرائيلية كونها الوحيدة المتوفرة وهذا ينطبق على 90 \% من الحوادث التي وقعت خلال أول أسبوعين منذ بدء الأحداث. ويعتقد أنه يجب أن يكون هناك استراتيجية واضحة للإعلام الفلسطيني تقوم على التحقق من كل معلومة قبل نشرها. كما يجب التحقق من كل حادثة على حدة ومحاولة التحقق من أي مصدر فلسطيني بعيداً عن الرواية الإسرائيلية.

الإعلام ليس 》ا يطلبه الجمهور "بل ما يجب أن يعرفه الجمهور • فالجمهور ذكي وحسه صادق دائما ولكن ردات فعله انفعالية بعيدة عن التعقل ولهذا تحتاج الحركات الجماهيرية إلى طليعة وقيادة والإعلام وسيط بين هذه الطليعة والجمهور • أن صحافة التحقق اليوم مهارة أساسية لا غنى عنها للصحافي والناشط المتابع لوسائل الإعلام الاجتماعي. بل إنها أصبحت مقدمة على النقد الإعلامي، لأن النقد يهتم بمضمون الرسالة وجودة التعبير عنها. أما التحقق فإنه يبحث في صحة المعلومة من الأصل. وقبل فهم وتجريب آليات التحقق لا بد من فهم الظروف التي أدت إلى تتامي الحاجة للتحقق الصحافي من أجل خلق فضول تحقق حميد وخلق تخيل أفضل مجالات الاستخدام. كما أن شدة التتافس على سرعة النشر التي تكون الدقة غالبا أولى ضحاياها. وسواء تمت التضحية بالدقة عن حسن نية او عن سوء نية فإن كثفها وتصحيحها واجب. وربما يستغل هؤلاء المتتافسون سخونة الاحداث واهتمام 


\section{Global Proceedings Repository \\ American Research Foundation}

ISSN 2476-017X

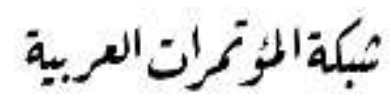

http://arab.kmshare.net/

Available online at http://proceedings.sriweb.org

الجمهور وتلكفه على الاخبار لتمرير أنباء كاذبة. وبشكل عام حيث يكون التسرع تكون الحاجة أكبر إلى التحقق وبشكل سريع من صحة ودقة المعلومات والصور •

أن المبالغة والتهويل سواء كانت بحسن نية او سوئها كثيرا ما تتم في عمليات النشر ـ استخدام الأوصاف

"كمسيرات حاشدة" او "مواجهات عنيفة" و "اشتباكات دموية" وغيرها من الأوصاف التهويلية دليل ضعف مهني ورغبة

في التضخيم. المحترفون لا يستعملونها ويستخدمون بدلا منها أعدادا تقديرية فيقولون مئات او آلاف او عشرات الألوف ويقولون اشتباكات بأسلحة.

ويبدو واضحاً أن الإعلام العبري متفوق على الإعلام الفلسطيني, لذلك هناك حالة إجماع لاى النخبة القليلة

من الصحافيين على وجوب إجراء مراجعة شاملة لمعايير الإعلام الفلسطيني وتثقيف الصحافيين للخروج من هذه الحالة التي تضر بصورة ومصداقية الإعلام المحلي، كما هو الحال بالنسبة للقضية الفلسطينية برمتها. 23

توظيف المواقع الاخبارية الاعلام الاجتماعي خلال أحداث "الهبة الثعبية"

في الوقت الذي يستمر فيه تقدم تكنولوجيا وسائط الإعلام وازدياد كثافة التدفق المعلوماتي وتعقد النظم الاجتماعية والسياسية والاقتصادية في العالم، وشعور الأفراد المتزايد كيانهم الذاتي وفاعليتهم كأفراد في بناء مجتمعاتهم تغيرت النظرة إلى تلك الوسائط، وظهر موقف جديد يعبر عن عهد فريد يتمثل في امتثاق الجيل الجديد من الثباب هذا "السلاح التقني".

هذا العصر الذي تزداد فيه دوائر الاتصال والتبادل اتساعا بغير حدود أو قيود، ويشارك فيه الثباب معاً في مناقشة المشكلات وإيجاد حلول لها، بل وإثارة مسائل وقضايا جديدة عن طريق تلك الثبكات التواصلية نجده واضح الأثر وعميق التأثير في الحالة الفلسطينية، تلك الحالة التي يثهد أبنائها هبة جماهيرية متراكمة لتاريخٍ طويل من النضال والتضحية والصمود.

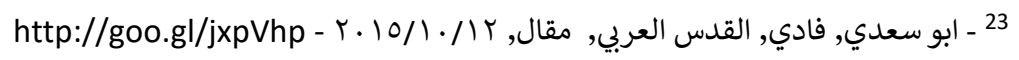




\section{Global Proceedings Repository \\ American Research Foundation}

ISSN 2476-017X

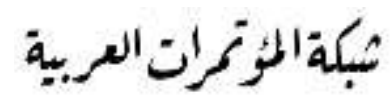

http://arab.kmshare.net/

Available online at http://proceedings.sriweb.org

هذه الهبة وغيرها استطاع الفلسطينيون أن يوظفوا فيها التقنيات الجديدة والمتطورة من وسائط الإعلام الجديد

(الإعلام الاجتماعي)، خاصة وسيلتي الفيسبوك واليوتيوب، بهدف فضح الممارسات الإسرائيلية بحق الثعب

الفلسطيني. هذه الوسائط التي استخدمها الثباب الفلسطيني بثكلٍ بارع ونافع أتاحت للعالم بأن يثاهد ويرى كم هي

الجرائم الفظيعة التي يمارسها الاحتلال الإسرائيلي بحق الإنسان والحياة الفلسطينية بثكل ممنهج ومتواصل.24

استخدام هذه التقنيات الإعلامية الجديدة طرحت في في الساحة الفلسطينية فكرة مفادها أن مظهراً جديداً

من "الناشطية” قد يمثل جزءاً لا يتجزأ من المجتمع المدني والمواطنين، أسلوبا مختلفا في تحديد الناشطة السياسية، وذلك مع نشوء الفضاء الافتراضي والتواصل الاككتروني، وهناك ثمة أشكال متتامية من النشاطات التي افتعلها

ابتكرها الثباب الفلسطيني عبر هذه الوسائط تم توظيفها في خدمة مواجهة المحتل وسياساته العدوانية والعنصرية .

هذه الثبكات الاتصالية والتواصلية، وخاصة ”الفيسبوك ” نجحت في نحت الوعي لاى الثباب الفلسطيني

وأثتتت مدى تأثيرها في وعي الإنسان وتصرفاته. ولعل السبب الأول في ذلك يعود إلى التقاعل مع الآخرين،

والاطلاع السريع على الأحداث والتتخل في الأزمات السياسية، بحيث شكلت وسائط الإعلام الجديدة عامل ضغط

كبير في أحداث كثيرة، استطاعت أن تتقل الحدث بصورته الواقعية والفورية والحقيقية إلى المجتمع الداخلي والعالم

دون تجزئه أو تشويش أو تعتيم، وهذا ما منح الفعل الفلسطيني وأشكال النشاط الوطني صدقية يتعاطى لها وبها كثير

$$
\text { من وسائل الإعلام في الغرب وشعوبه أيضاً. }
$$

الإعلام الفلسطيني والاجتماعي يفتقد الرؤية والاستراتيجية للتعامل مع الدعاية الإسرائيلية، مقابل عمل

الاخيرة وفق استراتيجية وخطط ممنهجة يشرف عليها خبراء، ويشارك فيها جيش من المتطوعين للحفاظ على صورة

الاسرائيلي كضحية والفلسطيني معتدي امام العالم، والتي كان آخرها تقديم 20 ألف إسرائيلي شكوى في محكمة

24 ـ فروخ, محمد, شبكة فلسطين الاخبارية, مقال, 0 


\section{Global Proceedings Repository \\ American Research Foundation}

ISSN 2476-017X

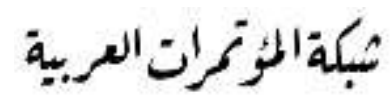

http://arab.kmshare.net/

Available online at http://proceedings.sriweb.org

امريكية ضد الفيسبوك لاعمه ما وصفوه بالتحريض وارتكاب الارهاب ضد الاسرائيليين على صفحات الفيسبوك الفلسطينية.

اسرائيل تعمل وفق منظومتين لتعزيز روايتها وتبرير جرائمها، الرواية الاولى الرسمية، وما يتبعها من صحف

وفضائيات وصفحات تواصل اجتماعي، وهي مجهزة تماما لإلصاق تهمة الإرهاب بالفلسطينيين وتصوير ما يقومون به من تصدى للاحتلال على أنه "أعمال إرهابية"، والمنظومة الثانية تتمثل فيما ينشره المتطرفون الذين يقومون بنشر الصور بعد كل عملية، بهدف تجريد الفلسطينيين من انسانيتهم وإظهارهم للمعتدين.

يمكن القول ان الاعلام الفلسطيني والاجتماعي فشل في التصدي للرواية الاسرائيلية امام العالم، باستثناء

حوادث قليلة مثل استشهاد الثاب بهاء عليان، والفيديو الذي وثق اطلاق النار على الطفل احد مناصرة، موضحا أنه "بعد نشر قصة الثهيد عليان الذي كان دخل موسوعة جينيس للأرقام القياسية بتثكيلة أطول سلسلة قراءة في مدينة القدس، فقد اثير الرأي العام حول السبب الذي يدفعه "لتنفيذ عملية" ادت الى انهاء حياته.

فان تصوير الثهيد كبطل وارفاق صور له أثناء تتفيذ العملية، وتتاقل رواية الاحتلال حول ما جرى، ساهم في تعزيز الدعاية الاسرائيلية امام العالم وافتقدنا التعاطف العالمي.26

ويفترض الابتعاد حين مخاطبة الجمهور المحلي والدولي عن الصور الدموية وان ننشر القصص الانسانية

والصور التي تجسد حياة الثهداء واحلامهم وطموحاتهم لإظهار حقيقة أن الضحية هو الفلسطيني الذي تحاول الدعاية الإسرائيلية تزييفها أمام العالم.

في هذه الهبة امام تراجعت الرواية الفلسطينية أمام الدعاية الاسرائيلية، مقارنة بالحرب على غزة، وذلك بفعل الماكنة الاعلامية والدعائية التي خصصتها اسرائيل لنشر روايتها حول ما يجري، عبر توزيع ونشر الأخبار والصور 


\section{Global Proceedings Repository \\ American Research Foundation}

ISSN 2476-017X

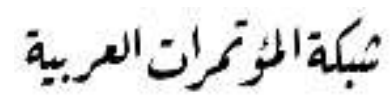

http://arab.kmshare.net/

Available online at http://proceedings.sriweb.org

والتقارير الممولة والمدعومة على صفحات التواصل الاجتماعي، وذلك بعكس الجانب الفلسطيني الذي تقوم نشاطاته على جهود فردية ومبادرات مبعثرة غير مدعومة في ظل غياب دعم حكومي للقيام بحملات مضادة.

تساهم في تعميق الفجوة بين القارئ والمشاهد والحدث، حيث تقوم كثير من الصفحات بتضخيم ما يحدث

وإظهار بطولات الثهداء للإبقاء على أجندات معينة والتحشيد، والحفاظ على صورة الأحداث القائمة واستمرارها. وحين يكتشف القارئ حقيقة ما جرى بأن الثهيد قد اعدم بدم بارد تتعمق الفجوة، يمل القارئ من متابعة ذلك، وهو من أصبحنا نلاحظه بشكل واضح من تراجع وانخفاض المشاركات والتفاعل على مواقع التواصل الاجتماعي حول

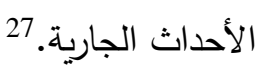

وكالة معا كمثال من المواقع الاخبارية الفلسطينية

انطلقت وكالة معا في عام O. . ب بدعم من المثليات للحكومة الهولندية والدنماركية في السلطة الوطنية الفلسطينية, وتعتبر من أكثر المواقع الفلسطينية زيارة الذي يزيد عن ثلاثة ملايين زائر شهريا, تقدم الأخبار على مدار الساعة باللغتين العربية والانجليزية, وهي احدى الوكالات القليلة التي لديها قسم متخصص بالثأن الإسرائيلي. وتغطي الوكالة الاخبار السياسية والاقتصادية والرياضية والثقافية لكافة محافظات الضفة الغربية وقطاع غزة عبر شبكة مراسلين, وتقوم باستمرار بترجمة مقتطفات من الصحافة العبرية الى اللغة العربية, ويتراوح عدد الاخبار يوميا

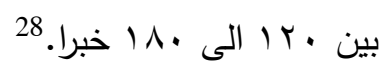
سياسة معا في التعامل مع الأخبار الواردة في الاعلام الاسرائيلي في العادة وكالة معا تتقل الرواية الثرطة الاسرائيلية في أحداث معينة تتعلق بعطليات فلسطينية تحدث في الداخل, في ظل شح المصادر الاخرى ولكن وكالة معا لا تاخذ وكالة رواية الشرطة الإسرائيلية كمسلمات لا من حيث الأرقام ولا من حيث الرواية وتفاصيل الرواية وأركان هذه الرواية. 


\section{Global Proceedings Repository \\ American Research Foundation}

ISSN 2476-017X

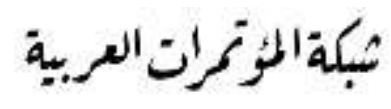

http://arab.kmshare.net/

Available online at http://proceedings.sriweb.org

تقوم وكالة معا في العادة الوسائل الاعلامية الفلسطينية الاخرى بايجاد رواية فلسطينية موازية مستندة للوقائع من حيث وجهة النظر الفلسطينية والجهة المنفذة سواء كانت فصيلا او منظمة او مجموعة فلسطينية او من خلال استتباط اراء الناس في القرية أو البلد الذي خرج منها منفذ العملية لموازاة رواية الثرطة الإسرائيلية. أما رواية الشرطة الاسرائيلية تأتي غالبا في ذيل الخبر الذي نشرته وكالة معا بالأولوية المطلقة حسب

الوكالة للرواية الفلسطينية ولكن يأتي فيها بعض ما ترده الشرطة الاسرائيلية لخلق بعض من التوازن والموضوعية لاطلاع الجمهور الاسرائيلي مع اعادة صياغة المصطلحات التي تستخدمها الشرطة الاسرائيلية واعادة التدقيق او اثارة التثكيك حول الأرقام التي تتشرها عبر طرح ارقام فلسطينية اخرى او عبر استخدام المصطلحات المعروفة في الإعلام مع الأرقام الواردة في الاعلام الاسرائيلي التي من خلالها يمكن التشكيك في الرواية الاسرائيلية.29 وكالة معا والمعلومات المتضلاربة الواردة في حينها يقول اللحام متخصص في الاخبار الاسرائيلية: "فيما يتعلق بالأحداث الساخنة وكالة معا بالعادة لا تلجأ الى مواقع التواصل الاجتماعي كمصدر للمعلومات" ويبرر ذلك أنه يمكن لهذه المعلومات أن تكون متضاربة ومتتاقضة قد تثير في بعض الأحيان الاضطرابات في المجتمع الفلسطيني. ويضيف اللحام أن وكالة معا يهمها في الأحداث الساخنة قبل الخبر او السبق الخبري دراسة هذا الخبر من حيث تأثيره ووقعه على المجتمع الفلسطيني حين نتحدث عن احداث ساخنة أو عن عمليات فلسطينية. وفي كيفية تعامل الوكالة مع الإسرائيليات والمعلومات المتضاربة يقول إن ذلك من خلال اتجاهين الأول: نشر هذه المعلومات من أكثر من مصدر متضارب حتى نضع الفلسطيني في صورة التثكيك وصورة التضارب في 


\section{Global Proceedings Repository \\ American Research Foundation}

ISSN 2476-017X

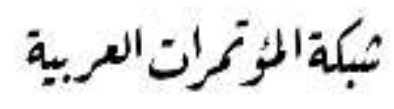

http://arab.kmshare.net/

Available online at http://proceedings.sriweb.org

الرواية الاسرائيلية وفي الاتجاه الاخر : تحاول ان توجد او ان تستنبط الرواية الفلسطينية التي ترد على هذا التضارب.30

في معا درجة من المهنية والتي يقول فيها المحرر السياسي لها ناصر عطية: " لا ندعي انها كبيرة او اننا أفضل من غيرنا الذي ربما نكون افضل من غيرنا, لكننا نتعامل بحذر ومهنية نوعا ما مع الإعلام الإسرائيلي وعدم أخذ ما يصدر عن الإعلام الإسرائيلي كما هو".

ويكمل: "العديد من الاخبار ننظر لها إنها مسربة من مخابرات او جهات امنية اسرائيلية مقصود فيها بث

آراء وبيانات موجهه بقصد من قبل المخابرات مثلا, وفي بعض الاحيان نرفض تتاول هذه المواضيع ولا نقوم بنشرها لدينا, وفي الأغلب تكون في الجانب السياسي والتي لا يكون فيها تصرف دقيق". وحول نقل التصريحات السياسية يقول عطية: "نأخذها كما هي لا يكون هنالك مجال للتلاعب فيها لكن في معلومات مختلفة اخرى ننظر فيها جيدا ونحن في قسم الترجمات في معا للاخبار الاسرائيلية لن تجد شبيها لها في موقع آخر, وكثير من الأخبار التي ننشرها على الوكالة سوف تجد اختلافا فيها عن المواقع الاخرى بشكل واضح, وليست كل الأمور نأخذها بحرفيتها في كل شيء تجدنا مدركين ما هي الأحداث التي يسعى ورائها الاعلام الاسرائيلي"البدائل أمام الإعلام الفلسطيني لاستبدال المصادر الاسرائيلية في العديد من الأحداث والقضايا التي يتتاولها الإعلام الفلسطيني لا يوجد داعي للعلم الاسرائيلي, فيوجد أحداث فلسطينية واعلام فلسطيني بكل تفاصيله, لكن هنالك مشكلة حادة في التعامل مع بعض القضايا التي تخضع لسلطة الاحتلال الاسرائيلي, بمعنى أن الصحفي الفلسطيني في معظم الأحيان لا يستطيع أن يصل للمصدر الحقيقي للمعلومة وغير قادر للوصول إلى المكان الذي يحصل به الحدث لذلك نجد أن في بعض الوسائل الإعلام تحاول أن تأخذ من المصادر الاسرائيلية, لكن يجب بالضرورة التعامل معها بحرفية عالية جدا والنظر للأهداف الاسرائيلية في نشر بعض المعطيات والحقائق وبعض القضايا في هذه الأخبار 


\section{Global Proceedings Repository \\ American Research Foundation}

ISSN 2476-017X

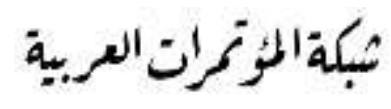

http://arab.kmshare.net/

Available online at http://proceedings.sriweb.org

قد يكون في بعض وسائل الإعلام الاجنبية كبديل لاى حضورها أثبت الاعلام الاسرائيلي انه السباق دائما للحدث كون سلطات الاحتلال موجودة في كل مكان وقادرة على فرض تعتيم وإغلاق المناطق وفقط الجانب الإسرائيلي هو من يصدر المعلومة.

ما يمكن القيام به هو ملاحقة أي حدث بطرق مختلفة عبر تدعيمها بوسائل اعلام اجنبية واذا توفرت مصادر محلية وشهود عيان او طرق مختلفة متوفرة اخرى لهذه القضايا للوصول الى حقيقة محددة حول العديد من الاخبار التي تتشرها وسائل الإعلام الاسرائيلية.31

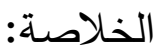
تخلص في هذه الدراسة إلى ما يلي:

- ان هناك ضرورة ملحة في صياغة رؤية اعلامية ترتقي برسالتها لتعزيز انسانية صورة الفلسطينيين. - التروي وتجنب اللحاق بأولوية السبق الصحفي, والتأكد من معرفة المعلومات المحيطة والمتفرعة من الحادثة, وإظهار المعلومات التي تم التأكد منها تماما من أكثر من مصدر بعيد وقوع الحدث. - ضرورة أن يكون هنالك حالة من التوازن ما بين نقل الواقع وبين عدم الوقوع في فخ ما يصدر من اخبار موجهه من الإعلام الإسرائيلي التي تكون عادة ذات مصدر أمني. - الابتعاد عن الترجمة المباشرة لما يصد عن الإعلام الاسرائيلي كما هو, وضرورة البحث عن ما وراء الاخبار التي

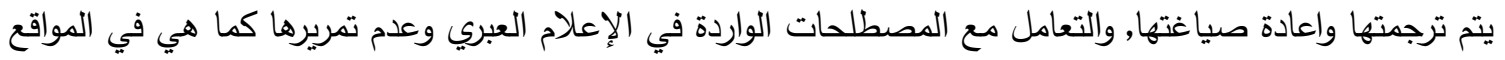
الاخبارية الفلسطينية.

- بالاضافة الى تجنب تسويق مواقف الحكومة الاسرائيلية التي تحرض بشكل ضمني على مدى طويل أو بشكل

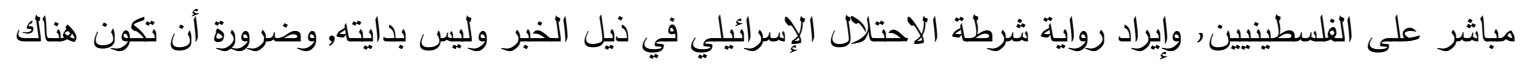
رواية فلسطينية في الخبر مع ما نقل عن رواية الاحتلال, وضرورة عدم ذكر رواية الاحتلال في الخبر لوحدها. - الابتعاد عن المبالغة والتهويل في المحتوى الاخباري وبث الوهن وتجنب خلق البلبلة. 


\section{Global Proceedings Repository \\ American Research Foundation}

ISSN 2476-017X

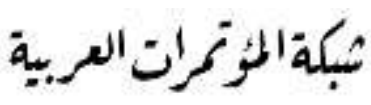

http://arab.kmshare.net/

Available online at http://proceedings.sriweb.org

- بالنسبة لاخبار الشهداء من الأفضل القيام بالاتصال بذوي الشهيد قبل ذكر اسمه, والتأكد بمعرفتهم بالخبر, والبعد عن استنطاق اهالي الثهداء بما يشبه عمل المحقق.

- إجراء المزيد من الدراسات حول الإعلام الفلسطيني ما بعد أوسلو وبالأخص المواقع الاخبارية الفلسطينية وتطورها

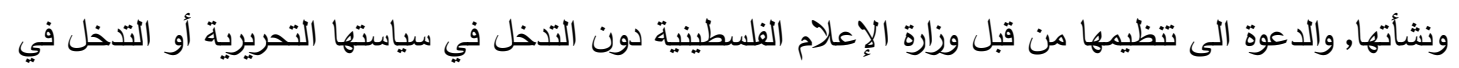
المضامين, ولكن التركيز على العمل ضمن قواعد المهنية الصحفية الصرفة.

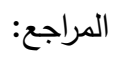

http://www.alquds.co.uk. 1

2.

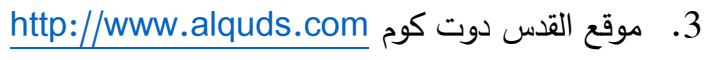

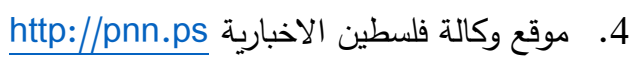

5. كتاب الإعلام الإسرائيلي مصطفى السعدي.

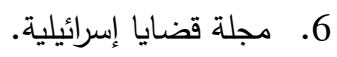

7. - . مجلة المشهد الاسرائيلي.

8. كتاب الموسوعة اليهودية.

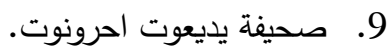

10. مدير قسم الاسرائيليات, وكالة معا الاخبارية.

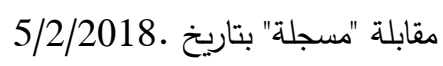

11. مسؤول قسم الإعلام الاجتماعي, وكالة معا الاخبارية.

مقابلة "مسجلة" بتاريخ .10/2/2018

12. محمد ضراغمة, متخصص في الإعلام الإسرائيلي.

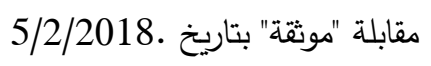

13. وزارة الإعلام الفلسطينية.

6/2/2018 مقابلة "موثقة" بتاريخ الإعائ

14. المحلل السياسي لوكالة معا الإخبارية.

مقابلة "موثقة" بتاريخ .7/2/2018

15. دراسة, الإعلام الإسرائيلي وانتفاضة الاقصى, عدنان أبو عامر .

16. دائرة سليمان الحلبي للدراسات الاستعمارية والتحرر المعرفي. 